\title{
Hyperinsulinemia Induces Insulin Receptor Dysfunction in Brain Microvascular Endothelial Cells
}

\author{
Luke S. Watson 1,2, Brynna Wilken-Resman 2, Alexus Williams 2, Guadalupe Sanchez 2, Taylor Lowry McLeod², \\ Catrina Sims-Robinson ${ }^{2, *}$ \\ 1 Medical University of South Carolina, Molecular and Cellular Biology and Pathobiology Program, Charles- \\ ton, South Carolina, 29425 USA \\ 2 Medical University of South Carolina, Department of Neurology, Charleston, South Carolina, 29425 USA \\ * Correspondence: Robinsoc@musc.edu; Tel.: (843) 792-0851
}

\begin{abstract}
Insulin receptors are internalized by endothelial cells; however, the impact of hyperinsulinemia on this process is not known. Thus, the aim of this study is to determine the role of hyperinsulinemia on insulin receptor function and internalization, as well as the potential impact of protein tyrosine phosphatase 1B (PTP1B). To this end, hippocampal microvessels were isolated from male $\mathrm{C} 57 \mathrm{Bl} / 6 \mathrm{~J}$ mice on either a control or high-fat diet and assessed for insulin receptor signaling. Cell surface insulin receptors in brain microvascular endothelial cells were labelled with biotin to assess the role hyperinsulinemia plays on receptor internalization in response to stimulation, with and without Claramine treatment, a potent PTP1B antagonist. Our results indicated that insulin receptor levels increased in tandem with insulin receptor dysfunction in the high-fat diet mouse hippocampal microvessels. Hyperinsulinemic cell-receptors demonstrate a shift in splice variation towards decreased IR-A/IR-B ratios and demonstrate a higher membrane-localized proportion. This corresponded with decreased autophosphorylation at sites critical for receptor internalization and signaling, however, Claramine restored signaling and receptor internalization in hyperinsulinemic cells. In conclusion, hyperinsulinemia negatively impacts brain microvascular endothelial cell insulin receptor function and internalization, likely through both alternative splicing and increased negative feedback from PTP1B.
\end{abstract}

Keywords: Blood-brain barrier, neurodegeneration, obesity, high-fat diet, protein tyrosine phosphatase- $1 b$

\section{Introduction}

Obesity impacts over 93 million adults in the United States and over 1 billion adults worldwide [1] , and leads to various metabolic complications including hyperglycemia, dyslipidemia, hypertension, and insulin resistance [2]. Obesity is also linked with an increased risk for developing cognitive impairment, neurodegenerative diseases, and brain atrophy [ $\underline{3-5}$ ], although the connection between this peripheral phenomenon and central nervous system functional decline is still an active area of investigation. An accumulating body of knowledge supports the role of sedentary lifestyle and the consumption of a diet with a high fat content in this pathogenesis [6,7]. A high-fat diet (HFD) leads to impaired learning and memory performance in animal models [ [-11]. Given the role obesity seems to be playing in memory impairment, this makes understanding the connecting mechanisms an important focus of research.

There are likely several factors which lead to obesity induced cognitive impairment, and recent focus has centered on endothelial dysfunction [5]. It is known that HFD leads to insulin resistance and hyperinsulinemia, a state of high circulating insulin within the plasma [12]. Insulin is a hormone released following feeding and its dysregulation is thought to contribute to the pathogenesis of diseases such as diabetes and Alzheimer's disease [13] . Insulin binds its receptor in the venous capillaries of the cerebral vasculature 
[1ㅡ], which is a selectively permeable, non-fenestrated endothelial layer $[\underline{15}, \underline{16}]$. Some point to the fact that hippocampal microvessels exhibit high concentrations of insulin receptors [17-19], coupled with the impact of insulin receptor signaling on cerebral blood flow, barrier integrity, and insulin transcytosis $[\underline{5}, \underline{20}, \underline{21}]$ as a potential underlying mechanism connecting obesity and memory decline. Much about the impact of obesity on the insulin receptor functioning at this unique barrier is still unknown.

Insulin binding to the insulin receptor leads to a conformational change, which allows for autophosphorylation of the receptor at specific tyrosine residues. This leads to the tyrosine phosphorylation of adaptor proteins such as the insulin receptor substrate (IRS)1, which in turn triggers signaling cascades such as phosphatidylinositol 3-kinase (PI3K) / protein kinase $\mathrm{b}(\mathrm{AKT})$ pathway $[\underline{22}, \underline{23}]$. While chronic serine phosphorylation of IRS $_{1}$ and reduced phospho-activated AKT have been indicated as markers of insulin resistance $[\underline{9}, \underline{4}]$, this ultimately stems from lack of insulin receptor autophosphorylation. This lack of insulin receptor autophosphorylation not only negatively impacts insulin receptor activation and downstream insulin signaling but also the internalization of the insulin/insulin receptor from the plasma membrane via endocytic vesicles [25-29]. This internalization plays a key role in regulating and sustaining insulin receptor signaling. Furthermore, some studies support the idea that internalization of insulin/insulin receptor is a necessary step in the transport of insulin into the brain via the brain microvascular endothelial cells [마]. There is a lack of information on how hyperinsulinemia impacts the internalization of the insulin receptor at this microvascular barrier.

Insulin receptor kinase activity and regulatory phosphatase crosstalk is essential for directing insulin receptor internalization and signaling [을. Increased tyrosine phosphatase activity, particularly protein tyrosine phosphatase 1B (PTP1B), is linked with impaired insulin receptor signaling and may contribute to pathological serine kinase activity associated with obesity $[\underline{24}, \underline{31}, \underline{32}]$. In fact, PTP1B is one of the major tyrosine phosphatases in endothelial cells. This prompted us to explore whether PTP1B leads to insulin receptor impairments during high-fat diet-induced hyperinsulinemia in microvascular endothelial cells.

In the present set of studies, we assessed insulin receptor signaling in isolated hippocampal microvessels, which represent the blood-brain barrier microenvironment including endothelial cells, from HFD mice. To address mechanisms, we utilized both mouse primary microvascular endothelial and human immortalized microvascular endothelial cells. Using molecular techniques, we assessed the internalization and phosphorylation state of the insulin receptor following experimental hyperinsulinemia and evaluated the potential role of PTP1B on insulin receptor internalization.

\section{Materials and Methods}

Animal Model

Three separate cohorts of five male C57BL6 mice, purchased from the Jackson Laboratory (Bar Harbor, Maine), were randomly assigned to either a standard diet consisting of $10 \%$ kcal from fat (STD; Research Diets Inc.; \#D12450B, New Brunswick, NJ) or a high-fat diet with 54\% kcal from fat (HFD; Research Diets Inc.; D05090701) ad libitum at approximately 5 weeks of age for 6 weeks.

Mice were housed in a pathogen-free environment. All protocols/procedures were approved by the Medical University of South Carolina Institutional Animal Care and Use Committee, and were conducted in accordance with the university guidelines, state and federal regulations, and the standards of the "Guide for the Care and Use of Laboratory Animals." Animal Welfare Assurance Number on file with the NIH Office of Laboratory Animal Welfare (OLAW) is A3428-01 (Medical University of South Carolina). The Medical University of South Carolina is accredited by the Association for the Assessment and Accreditation of Laboratory Animal Care International (AAALAC, Intl.). 


\section{Pooled Hippocampal Microvessel Isolation}

Microvessels were isolated per previous protocols $[\underline{33}, \underline{34}]$ with minor modifications. Briefly, after 4 hours of fasting, mice on either a STD or HFD were euthanized via cervical dislocation. Pooled hippocampal samples from five mice were transferred to a $50 \mathrm{ml}$ Falcon tube filled with 2.5-5 ml of 1x PBS containing protease inhibitor (Roche, Branchburg, NJ). The tissues were minced with a 18, 23, and finally 25-gauge needle/syringe until the media became milky. Cells were then centrifuged at $4000 \times \mathrm{g}, 4^{\circ} \mathrm{C}$ for 10 minutes, and the supernatant was removed. Pellets were re-suspended in 30\% Dextran (31390, Sigma-Aldrich Burlington, MA) approximately 25 times at 1:3 pellet:Dextran ratio, and re-centrifuged at $10,000 \times \mathrm{g}, 4^{\circ} \mathrm{C}$ for 10 minutes. The myelin layer and supernatant were discarded, and the pellet was re-suspended in $50 \mu \mathrm{l}$ 1x PBS. Microvessels were then passed through a cell strainer (40 $\mu \mathrm{m}$, Greiner Bio-one, Monroe, North Carolina) with 500ul 1x PBS and treated with $20 \mathrm{nM}$ insulin or saline for 15 minutes diluted in the $1 \mathrm{x}$ PBS, centrifuged at $10,000 \times \mathrm{g}, 4^{\circ} \mathrm{C}$ for 5 minutes. The supernate was discarded and the pellet was re-suspended in $300 \mu \mathrm{l}$ T-PER with protease inhibitor for analysis. The samples were stored at $80^{\circ} \mathrm{C}$ until use for western immunoblotting as described below.

\section{Microvascular Endothelial Cell Culture}

Primary C57BL/6 mouse brain microvascular endothelial cells (MBECs; C57-6023, Cell Biologics, Chicago, IL) were seeded in 12-well plates coated with $5 \mu \mathrm{g} / \mathrm{cm}^{2}$ rat tail collagen type I (A1048301, Gibco, Waltham, MA) for cell assays and allowed to grow to 80-90\% confluency before treatment. Cells were grown in Complete Mouse Endothelial Cell Medium with supplemental kit (M1168 + kit, Cell Biologics), at $37^{\circ} \mathrm{C}$ in $5 \% \mathrm{CO}_{2}$. All experiments were completed on cell passage 5 .

Human cerebral microvascular endothelial cells were isolated from post-mortem brains of donors and transfected with plasmid containing SV40 large T antigen (HBEC-5i; CRL-3245 ${ }^{\mathrm{TM}}$, ATCC ${ }^{\circ}$, Manassas, VA). Cells were grown in F12:DMEM plus 10\% FBS and Endothelial Cell Growth Supplement (356006, Corning) on 0.1\% Gelatin (PCS-999-027, ATCC®). To exclude the effect of growth factors, culture medium was changed to F12:DMEM without serum 4 hours prior to treatment. All experiments were completed on cell passage 4 .

All insulin stimulation and inhibitor treatments were performed at $37^{\circ} \mathrm{C}$. Hyperinsulinemic conditioning was induced in MBECs by incubating cells for 12 hours in 20nM human recombinant insulin (19278, Sigma, St. Louis, MO), and HBEC-5is in $5 \mathrm{nM}$ human recombinant insulin for 12 hours. Time and concentrations for hyperinsulinemic conditioning were determined in pilot studies, data not presented here.

\section{Cell Assays and Analysis Preparation}

Human recombinant insulin and $1 \mathrm{ug}$ total of Claramine trifluoroacetate (SML1545, Sigma-Aldrich) were diluted directly into culture media. Prior to treatment, cells were washed with warm 1x PBS solution (Corning ${ }^{\mathrm{TM}}$ Mediatech $^{\mathrm{TM}}$, Tewksbury, MA) and replaced with fresh media for at least 30 minutes. After treatment, media was removed and supplanted with T-PER (Thermo Scientific ${ }^{\mathrm{TM}}$, Waltham, MA) + protease inhibitor (Roche), scraped, and stored at $-80^{\circ} \mathrm{C}$ until analysis.

\section{Cell Surface Receptor Biotinylation}

Cell surface receptor biotinylation was performed as previously described $[\underline{35}, \underline{36}]$. Briefly, following hyperinsulinemic conditioning, cells were washed with cold 1xPBS, and incubated in $0.5 \mathrm{mg} / \mathrm{ml}$ EZ-Link ${ }^{\mathrm{TM}}$ Sulfo-NHS-SS-Biotin (21331, ThermoFisher Scientific, Waltham, MA) for 30 minutes at $4^{\circ} \mathrm{C}$. Cells were immediately quenched with glycine- $\mathrm{HCl}$, aspirated, and supplanted with $250 \mu \mathrm{L}$ of T-PER + protease inhibitor and collected. Lysates were incubated at room temperature for 1 hour in Streptavidin Plus UltraLink ${ }^{\mathrm{TM}}$ Resin (Pierce ${ }^{\mathrm{TM}}$, Waltham, MA), followed by centrifugation at $400 \mathrm{x} \mathrm{g}$ for 10 minutes. 
Supernates, which contain the non-biotinylated (i.e. internalized) receptor pool, were collected. To remove the biotinylation, the pellet was treated in glycine- $\mathrm{HCl}$ and vortexed for 10 seconds, followed by 10 minutes of incubation at room temperature. Cells were centrifuged at 4,000 x g for 10 minutes, and supernates were collected for analysis.

\section{Stimulation Induced Biotinylated Receptor Internalization}

Biotinylated receptor internalization analysis was performed as previously described $[\underline{35}, \underline{36}]$. Briefly, following hyperinsulinemic conditioning, cells were washed with cold 1xPBS, and incubated in $1 \mathrm{mg} / \mathrm{ml}$ EZ-Link ${ }^{\mathrm{TM}}$ Sulfo-NHS-SS-Biotin (21331, ThermoFisher Scientific, Waltham, MA) for 15 minutes at $4^{\circ} \mathrm{C}$. Cells were immediately washed in cold 1xPBS, and incubated in complete medium containing $20 \mathrm{nM}$ recombinant human insulin for the designated period of time. Cells were immediately washed in cold 1xPBS. Cells were then incubated in a glutathione buffer for 10 minutes followed by a rinse in cold 1xPBS. This buffer wash was repeated 3 times in order to cleave extracellular biotin. Finally, cells were supplanted with $200 \mu \mathrm{L}$ of T-PER + protease inhibitor and collected. A portion of the sample was saved for total protein analysis and western immunoblotting. The rest of the lysates were incubated at room temperature for 1 hour in Streptavidin Plus UltraLink $^{\mathrm{TM}}$ Resin (Pierce $^{\mathrm{TM}}$, Waltham, MA), followed by centrifugation at $400 \times \mathrm{g}$ for 10 minutes and removal of the supernates. The pellet was resuspended in 1x PBS + protease inhibitors, ready to be processed for protein analysis.

\section{Protein Analysis}

Lysate preparation

Hippocampal microvessel samples were analyzed for utilizing the Pierce Protein Assay for protein concentration quantification in order to ensure equal sample concentration. Samples were subjected to sodium dodecyl sulfate (SDS) to make lysates for polyacrylamide electrophoresis and western immunoblotting analysis.

\section{Western immunoblotting}

Western immunoblotting was performed per our previously published protocol [37]. Briefly, lysates were separated by SDS-PAGE and transferred to a nitrocellulose membrane. TBS with Tween-20 supplemented with 5\% BSA was used to block the membranes and to dilute the antibodies. Polyclonal antibodies against protein kinase B (AKT), phos-

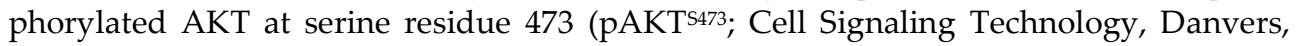
$\mathrm{MA}$ ), and tubulin (Abcam, Cambridge, MA), were used at a 1:1,000 dilution. Insulin receptor substrate (IRS)1, IRS 1 phosphorylated at the serine residue 307 ( $\mathrm{pIRS}_{1}$ S307; $_{\text {; }}$ equivalent to human pIRS $\left._{1}{ }^{5312}\right)$, insulin receptor (InsR- $\beta$ ), phosphorylated insulin receptor at tyrosine residues 1150/1151 (pInsR ${ }^{\mathrm{Y} 1150 / 1151}$; all from Cell Signaling Technology), were used at a 1:750 dilution. The appropriate horseradish peroxidase-conjugated secondary antibodies (Cell Signaling Technology) was used at a 1:2,000 dilution. Protein signal quantification was visualized using Clarity Western ECL Substrate (Bio-Rad Laboratory, Hercules, CA), with images captured using ChemiDoc ${ }^{\mathrm{TM}}$ Imaging System, analyzed by Image Lab software (Bio-Rad Laboratory), and normalized to total lane protein concentrations.

\section{Receptor Splice Variant Analysis}

Total RNA was isolated from cells using the RNeasy mini kit (74104, Qiagen, Germantown, MD) and was reverse transcribed using QuantiTect Reverse Transcription Kit (205311, Qiagen) per the manufacturer's instructions from $1 \mu \mathrm{g}$ of total RNA. Quantitative PCR (qPCR) analysis was performed using the CFX Connect qPCR system (Bio-Rad Laboratory) with primers described in Table 1 (validated in a previous publication [38]). Relative quantification of gene expression was calculated by the comparative threshold cycle $(\mathrm{Ct})$ method and expressed as $2^{(-\Delta \Delta \mathrm{C} t)}$, using GADPH gene as an internal control. 


\section{Statistical Analysis}

Data analyses were performed using Prism v9 (GraphPad Software, Inc., San Diego, CA). Data was quality checked for outliers using the ROUT outlier analysis $(Q=1 \%)$ prior to performing statistical analyses. All data was analyzed via two-tailed $\mathrm{t}$-test with two exceptions. First, the MBEC InsR- $\beta$ internalization after 15 minutes of stimulation experiment was analyzed using a one-way analysis of variance (ANOVA) with Tukey's multiple comparisons test. Finally, the supplemental InsR- $\beta$ internalization time course experiment was analyzed via two-way ANOVA with Bonferroni's multiple comparisons test. All data is represented as mean \pm standard error of the mean, and significance was determined using an alpha-level of 0.05 . Cell culture sample sizes represent the number of total biological replicates $(\mathrm{n})$ over the number of independent experiments $(\mathrm{N})$.

\section{Results}

Insulin receptor levels are elevated and exhibit impaired signaling in hippocampal microvessels from high-fat diet mice

We first sought to assess the impact of diet on insulin receptor levels and function in the hippocampal microvessels from STD and HFD mice ( $n=3$ / group). Microvessels from HFD mice demonstrate an increase of $118 \% \pm 37 \%$ in total insulin receptor levels (Figure $1 \mathrm{~A}-\mathrm{B})$ compared to microvessels from STD mice $(\mathrm{t}(4)=3.174, \mathrm{p}=0.0337)$. Following ex vivo stimulation with $20 \mathrm{nM}$ of insulin for 15 minutes, microvessels from HFD mice demonstrate a decrease of $132 \% \pm 37 \%$ in $\mathrm{pAKT}^{\mathrm{s} 473} / \mathrm{AKT}$ levels compared to stimulated microvessels of STD mice ( $t(3)=3.896, p=0.0176$; Figure 1C-D).

A

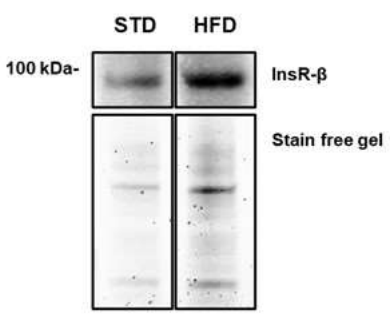

C

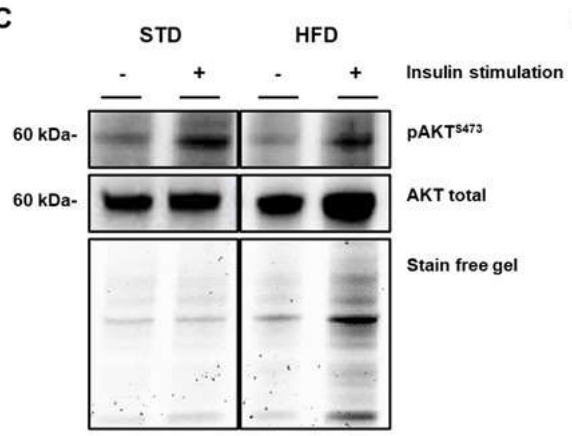

B

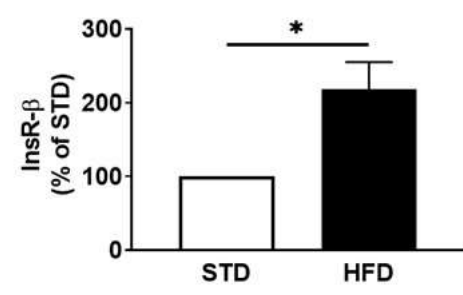

D

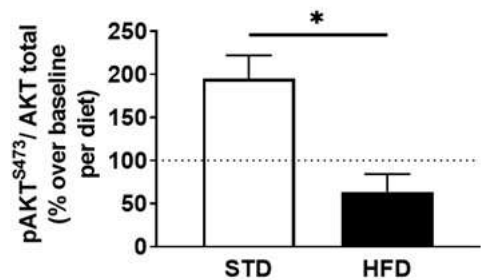

Figure 1. Hippocampal microvessels isolated from high-fat diet mice exhibit signs of insulin receptor dysfunction. (A) Immunoblot and (B) densitometry analysis of insulin receptor (InsR- $\beta$ ) in hippocampal microvessels isolated from mice after 6 weeks of either a standard diet (STD) or a high-fat diet (HFD). (C) Immunoblot and (D) densitometry analysis of protein kinase B (AKT) phosphorylated at serine 473 (pAKT ${ }^{5473}$ ), and total AKT following ex vivo stimulation with $20 \mathrm{nM}$ insulin for 15 minutes in microvessels isolated from STD and HFD mice. There were 5 pooled mouse hippocampi per experiment, $n=3$. All images were normalized to total gel protein concentrations shown as "Stain free gel", calculated in Image Lab software per the manufacturer's instructions. Line down center of immunoblot indicates a break in the blot; images are from the same immunoblot for each figure and received identical formatting. 
Insulin receptor levels are elevated and exhibit impaired insulin signaling in brain endothelial cells following experimental hyperinsulinemia

Given that microvessels contain multiple cell types, we assessed the impact of hyperinsulinemia on insulin receptor levels and function in primary mouse brain endothelial cells (MBECs; $\mathrm{n}=9-12$ / group, $\mathrm{N}=4$ ). Indeed, MBECs treated with experimental hyperinsulinemia demonstrate a significant increase of $263 \% \pm 78 \%$ in total insulin receptors compared to untreated MBECs $(\mathrm{t}(21)=3.384, \mathrm{p}=0.0028$; Figure $2 \mathrm{~A}-\mathrm{B})$. Further, analysis of the IRS $_{1}$ protein demonstrates that hyperinsulinemic treatment of MBECs exhibit a significant increase in serine phosphorylation by $281 \% \pm 92 \%$ compared to untreated MBECs $(\mathrm{t}(19)=$ 3.043, $\mathrm{p}=0.0067$; Figure 2A-C).

We also confirmed these findings in human cerebral microvascular endothelial cells (HBEC-5is, $\mathrm{n}=7-10, \mathrm{~N}=4$ ). HBEC-5is treated with experimental hyperinsulinemia demonstrate a significant increase of $288 \% \pm 135 \%$ in total insulin receptors compared to untreated HBEC-5is $(t(16)=2.129, p=0.049$; Figure $2 C-D)$. Further, analysis of the IRS 1 protein demonstrates that hyperinsulinemic treatment of HBEC-5is exhibit a significant increase in serine phosphorylation by $281 \% \pm 92 \%$ compared to untreated HBEC-5is $(\mathrm{t}(12)=$ 3.439, $\mathrm{p}=0.0044$; Figure 2C-D).

A

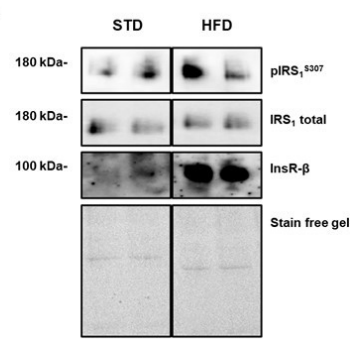

D

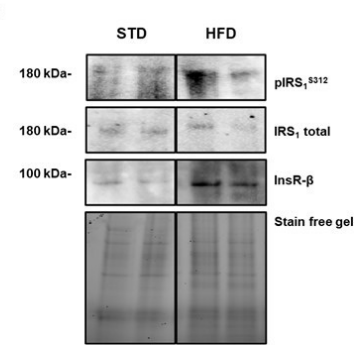

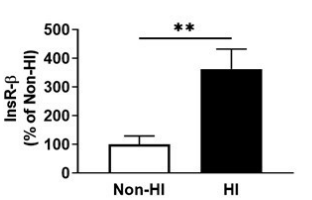

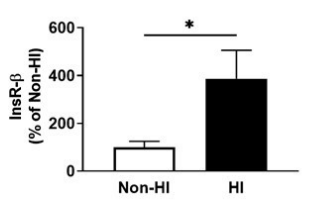

C

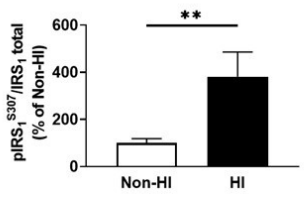

$\mathbf{F}$

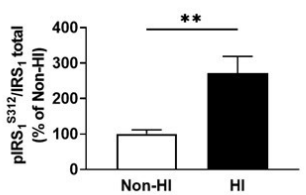

Figure 2. Experimentally hyperinsulinemic microvascular brain endothelial cells exhibit dysfunctional insulin receptors. (A) Immunoblots and densitometry analysis for (B) total insulin receptor (InsR- $\beta$ ), and (C) insulin receptor substrate 1 (IRS) 1 phosphorylated at the 307 residue $\left(\mathrm{pIRS}_{1}{ }^{\mathrm{S} 307}\right) / \mathrm{IRS}_{1}$ total, in mouse brain microvascular endothelial cells (MBECs) that are untreated (non-HI) or treated in hyperinsulinemic conditions for 12 hours (HI). (D) Immunoblots and densitometry analysis for (E) total InsR- $\beta$, and $(\mathrm{F}) \mathrm{IRS}_{1}$ phosphorylated at the 312 residue ( $\mathrm{pIRS}_{1}{ }^{\mathrm{S} 312}$; the human equivalent of mouse S307)/ IRS 1 total, in non-HI or HI human immortalized brain microvascular endothelial cells (HBEC-5is).

Data was analyzed via two-tailed t-test, $\mathrm{n}=9-12$ / group, $\mathrm{N}=4$ (MBECs) and $\mathrm{n}=7-10, \mathrm{~N}=4$ (HBEC5 is), $* \mathrm{p}<0.05, * * \mathrm{p}<0.01$. All images were normalized to total gel protein concentrations shown as "Stain Free Gel", calculated in Image Lab software per the manufacturer's instructions. Line down center of immunoblot indicates a break in the blot; images are from the same immunoblot for each figure and received identical formatting.

\section{Hyperinsulinemia leads to differential receptor splice variation}

We next sought to examine whether hyperinsulinemia had an impact on receptor splice variation through RT-qPCR quantification of both receptor isoforms A (IR-A) and B (IR-B). Our data indicate that MBECs treated with experimental hyperinsulinemia demonstrate a significant increase of $79 \% \pm 7 \%$ in IR-A levels compared to untreated 
MBECs $(t(14)=11.94, p<0.0001 ;$ Figure 3B), however there were no significant differences in IR-B levels (Figure 3C). This translated to a significant decrease of $54 \% \pm 11 \%$ in IR-A/ IR-B ratios $(t(12)=4.966, p=0.0003$; Figure $3 \mathrm{D})$ and a significant overall decrease of $70 \%$ $\pm 10 \%$ in total insulin receptors $(t(11)=7.288, p<0.0001$; Figure 3E) for MBECs treated with experimental hyperinsulinemia compared to untreated MBECs.

Table 1. Primers used for experiments. Primers sequences for splice variants were chosen for inclusion or exclusion of the junction between exon 10 and exon 12 (see Figure 5A). Splice variant specific primers were created based off a previous publication (see Supplementary Appendix in [38]), GADPH primers were ordered off of Integrated DNA Technologies ReadyMade ${ }^{\mathrm{TM}}$ Primers site: https://www.idtdna.com/pages/products/custom-dna-rna/readymade-inventoried-oligos/readymade-primers.

\begin{tabular}{cc}
\hline Primer & $\mathbf{5}^{\prime} \rightarrow \mathbf{3}^{\prime}$ \\
\hline GADPH Forward & ACC ACA GTC CAT GCC ATC AC \\
GADPH Reverse & TCC ACC ACC CTC TTG CTG TA \\
IR-A/B Forward & TCC TGA AGG AGC TGG AGG AGT \\
IR-A Reverse & CTT TCG GGA TGG CCT GG \\
IR-B Reverse & TTC GGG ATG GCC TAC TGT \\
\hline
\end{tabular}

A

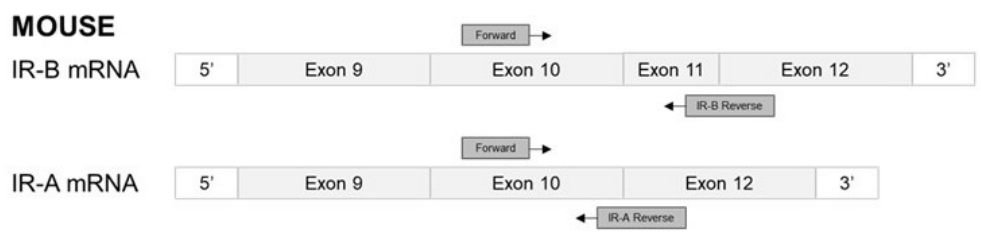

B

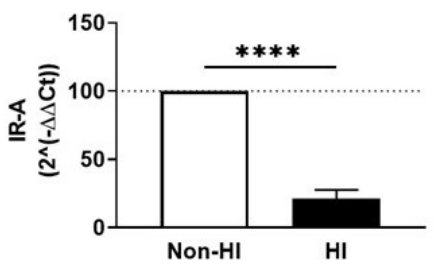

D

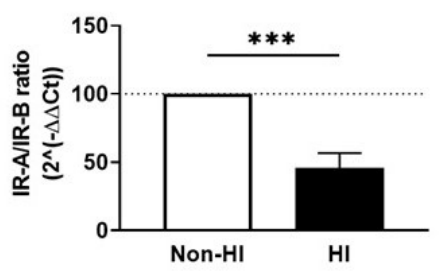

C

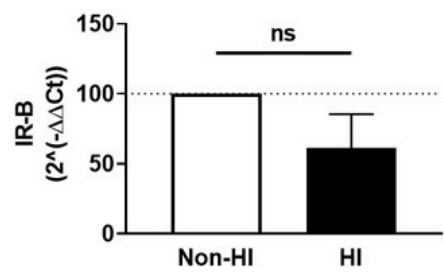

E

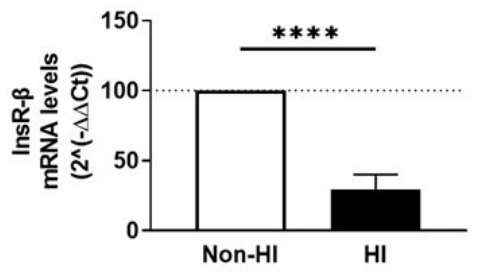

Figure 2. Diagram of RT-qPCR primer design for insulin receptor isoforms A and B (IR-A and IR-B, respectively), using exons 10-12 of the mouse insulin receptor coding region. Primers (Table 1) were designed specifically for each isoform. Calculated $2^{(-\Delta \Delta C t)}$ values for (B) IR-A and (C) IR-B in mouse brain microvascular endothelial cells (MBECs) that are untreated (non-HI) or treated with experimental hyperinsulinemic conditions for 12 hours (HI), normalized to non-HI. (D) Ratio of the $2^{\text {(- }-\Delta \Delta \mathrm{Ct})}$ values for IR-A and IR-B variants in non-HI and HI MBECs, normalized to non-HI. (C) Total insulin receptor mRNA levels in non-HI and HI MBECs, normalized to non-HI.

(B-E) Data analyzed via two-tailed t-test between non-HI and HI MBECs, n= 6-7/ group, N=4; $* * * \mathrm{p}<0.001, * * * * \mathrm{p}<0.0001$. GADPH was used as internal control for all RT-qPCR experiments.

Insulin receptor internalization is reduced following hyperinsulinemia in MBECs 
Given that the insulin receptor isoform may impact receptor internalization, we assessed the insulin receptor internalization in MBECs $(n=6-9 /$ group, $N=3-4)$. The analysis indicates a significant effect of time $(F(3,52)=3.501, p=0.0217)$ and treatment $(F(1,52)=$ $7.175, p=0.0099)$. A Bonferroni's multiple comparison revealed significant internalized insulin receptors at 15 minutes $(p=0.0342)$ and 30 minutes $(p=0.0136)$ after stimulating with insulin in untreated MBECs. There were no changes to insulin receptor internalization in the MBECs treated with hyperinsulinemia at any timepoint (Supp. Figure 1).

We then sought to understand how this impacted downstream processes of the insulin receptor. MBECs treated with experimental hyperinsulinemia display a significant increase of $384 \% \pm 162 \%$ in membrane insulin receptor levels of compared to untreated MBECs $(t(12)=2.373, p=0.0352$; Figure 4A-B). To assess downstream signaling, non-hyperinsulinemic and hyperinsulinemic cells were stimulated with 20nM insulin for 15 minutes to probe for autophosphorylation of the insulin receptor and downstream AKT phosphorylation. Following stimulation, MBECs treated with experimental hyperinsulinemia display a significant decrease of $117 \% \pm 29 \%$ in phosphorylated insulin receptor/ total insulin receptor compared to untreated MBECs, normalized to non-stimulated control groups for each $(\mathrm{t}(14)=4.085, \mathrm{p}=0.0011$; Figure $4 \mathrm{C}-\mathrm{D})$. This coincided with a significant decrease of $106 \% \pm 48 \%$ in phosphorylated AKT/ total AKT compared to untreated MBECs, normalized to non-stimulated control groups for each $(t(12)=2.188, p=0.0492$; Figure 4E-F).

A

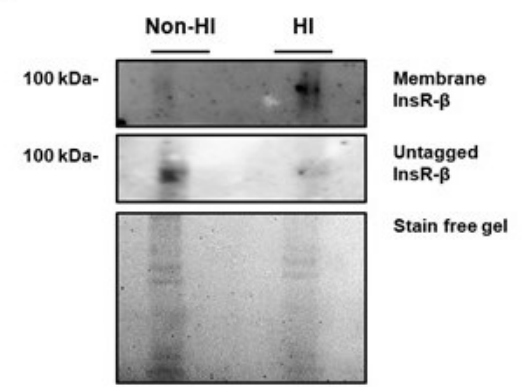

C

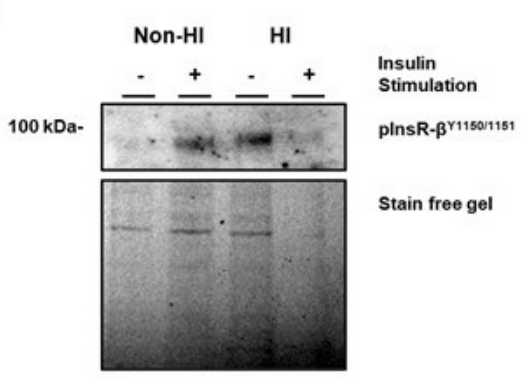

E

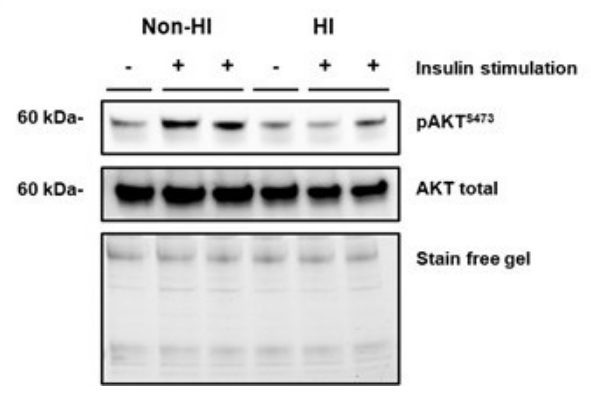

B

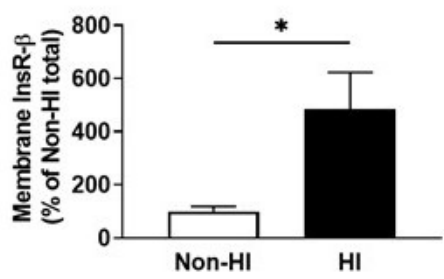

D

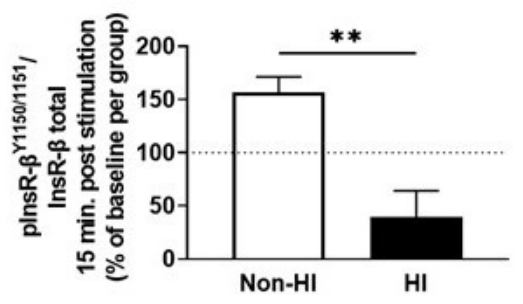

F

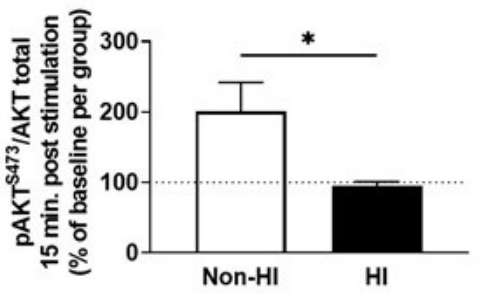

Figure 4. Despite having increased insulin receptor levels, hyperinsulinemic MBECs do not stimulate downstream signaling via tyrosine autophosphorylation. (A) Immunoblots and (B) densitometry analysis for insulin receptor (InsR- $\beta$ ) tagged on the membrane and untagged InsR- $\beta$ mouse brain microvascular endothelial cells (MBECs) treated with experimental hyperinsulinemic 
conditions for 12 hours (HI), and untreated MBECS (non-HI). Data represented as \% of total InsR- $\beta$ per group. (C) Immunoblot and (D) densitometry analysis for tyrosine phosphorylated InsR- $\beta$ at the 1150/1151 residues (pInsR- $\beta^{\mathrm{Y} 1150 / 1151}$ ). (E) Immunoblots and (F) densitometry analysis for protein kinase B (AKT) phosphorylated at serine residue 473 (pAKT ${ }^{5473}$ ) and AKT total following $20 \mathrm{nM}$ insulin stimulation for 15 minutes.

Data was analyzed via two-tailed t-test, $\mathrm{n}=6-9 /$ group, $\mathrm{N}=3-4 ; * \mathrm{p}<0.05, * * \mathrm{p}<0.01$. All images were normalized to total gel protein concentrations shown as "Stain Free Gel", calculated in Image Lab software per the manufacturer's instructions.

Selective inhibition of PTP1B improves hyperinsulinemia-induced insulin receptor function and internalization

Given the decrease in tyrosine phosphorylation, we next sought to assess whether Claramine, a selective PTP1B inhibitor, could reverse the effects of MBECs treated with hyperinsulinemia when stimulated with insulin for 15 minutes $(n=9-12, N=4)$. Treatment of hyperinsulinemic MBECs with Claramine prior to insulin stimulation led to a significant increase of $143 \% \pm 61 \%$ in phosphorylated AKT/ total AKT compared to untreated hyperinsulinemic MBECs, normalized to the non-stimulated hyperinsulinemic $(\mathrm{t}(18)=$ 2.418, $\mathrm{p}=$ 0.0264; Figure 5A-B). When we probed for internalization capacity following stimulation, we demonstrate a significant difference in internalization between untreated MBECs, MBECS treated with hyperinsulinemia, and MBECs treated with hyperinsulinemia and Claramine $(F(1,17)=3.921, p=0.0398)$. Tukey's post-hoc comparison indicates increased internalization between untreated MBECs and MBECS treated with hyperinsulinemia by $359 \% \pm 133 \%$ ( $p=0.0380$; Figure $5 C-D)$.

A

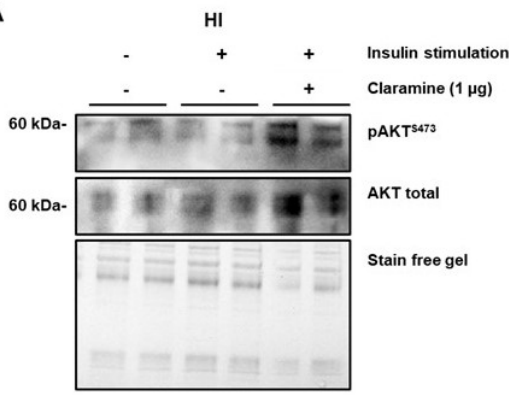

C

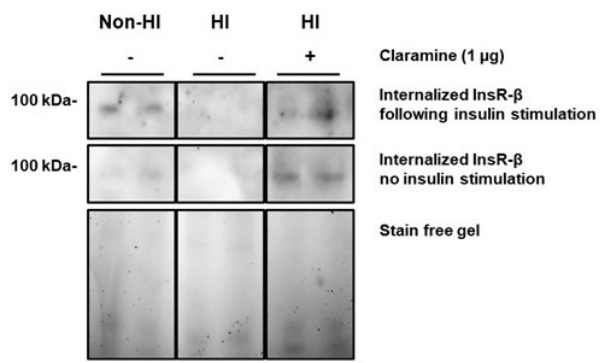

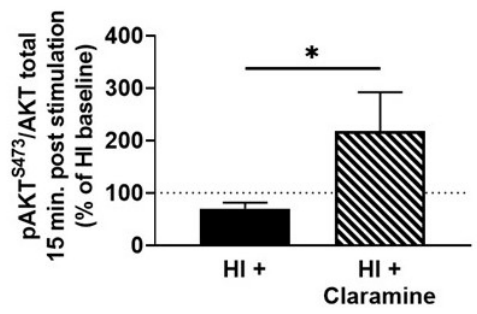

D

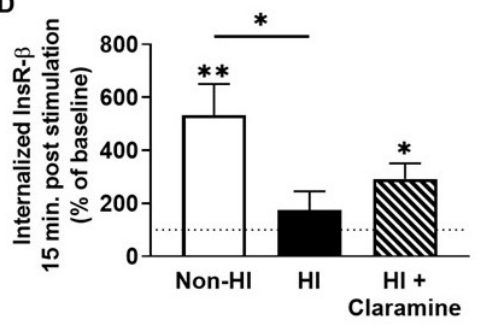

Figure 5. Claramine treatment restores insulin receptor signaling and improves receptor internalization. (A) Immunoblots and (B) densitometry analysis for protein kinase B (AKT) phosphorylated at serine residue $473\left(\mathrm{pAKT}^{\mathrm{S} 473}\right)$ at baseline, $\mathrm{pAKT}^{\mathrm{S473}}$ after 15 minutes of $20 \mathrm{nM}$ insulin stimulation, and AKT total in mouse brain microvascular endothelial cells (MBECs) treated with just experimental hyperinsulinemic conditions for 12 hours (HI) or HI pre-treated for 30 minutes before stimulation with $1 \mu \mathrm{g}$ of Claramine (HI + Claramine). (C) Immunoblots and (D) densitometry for internalized insulin receptor beta (InsR- $\beta$ ) at baseline and internalized InsR- $\beta$ after 15 minutes of $20 \mathrm{nM}$ insulin stimulation in untreated MBECs (non-HI), HI MBECs, and HI + Claramine MBECs.

(A-B) Data was analyzed via two-tailed t-test, $\mathrm{n}=8-12$ / group, $\mathrm{N}=4 ; * \mathrm{p}<0.05$. (C-D) Data analyzed via a two-tailed t-test to group baselines and a one-way ANOVA with Tukey's multiple comparisons, $\mathrm{n}=6-8$ / group, $\mathrm{N}=4$. All images were normalized to total gel protein concentrations shown as "Stain Free Gel", calculated in Image Lab software per the manufacturer's instructions. Line down 
center of immunoblot indicates a break in the blot; images are from the same immunoblot for each figure and received identical formatting.

\section{Discussion}

Microvascular dysfunction is a potential underlying mechanism linking obesity and cognitive impairment that has garnered more attention over the past decade. The brain microvasculature is a unique subset of the vascular system known as the blood-brain barrier which maintains relative exclusivity of the brain from circulating substances[5]. The brain microvascular endothelial cells play a significant role in mediating the transport of hormones and molecules across the blood-brain barrier. For example, insulin must cross the blood-brain barrier for delivery to tissues such as the hippocampus for normal function and signaling. It is well known that diet-induced hyperinsulinemia leads to a decrease in the transport of insulin across the blood brain barrier [ $\underline{21}, \underline{39}, \underline{40}]$. Currently, it is controversial how this transport occurs, whether it is via a paracellular route [41] or via internalization of the insulin/insulin receptor. Hence, the goal of this current study was to understand the impact obesity plays on insulin receptor functioning within the brain microvascular endothelium. Our primary findings indicate that high-fat diet leads to increased total insulin receptor levels in brain microvasculature, which exhibit impaired signaling post-stimulation. We replicated this increase in insulin receptor levels and impaired signaling with experimentally hyperinsulinemic cultured brain endothelial cells in vitro. Upon further analysis, we revealed that this corresponded with a shift in insulin receptor splice variation and increased membrane presentation of insulin receptors but decreased the ability for receptors to internalize and to activate signaling cascade effectors via tyrosine autophosphorylation. Signaling and internalization were both improved via selective inhibition of PTP1B.

Membrane receptor tyrosine kinases such as the insulin receptors are unique in their ability to stimulate growth signaling and maintenance of homeostasis [느]. Insulin signaling plays an important role in regulating endothelial cell function. Indeed, multiple studies have associated brain microvascular endothelial cell insulin receptor functioning with regulation of cerebrovascular blood flow via stimulation of nitric oxide and intracellular calcium $[\underline{5}, \underline{3}]$. Patients with hyperinsulinemia-induced insulin resistance and preclinical models alike demonstrate impaired hippocampal health and functioning $[\underline{4}, \underline{9}, \underline{4}, \underline{45}]$. Given that the hippocampus, which is intimately involved with memory processing, displays dense insulin receptor expression along its vasculature [17-19], there may be an underlying connection. Thus, we sought to investigate the impact of diet-induced hyperinsulinemia on the microvasculature. First, our data in pooled hippocampal microvessels from high-fat diet fed mice showcase increased levels of total insulin receptors (Figure 1). This is surprising given that a previous report by Gray, Aylor \& Barrett [으] isolated microvessels from eight week-old rats on a similar dietary treatment for four weeks demonstrated no such difference. This discrepancy may be explained by important methodological differences. For example, we isolated microvessels specifically from the hippocampus, whereas the former study isolated endothelial cells from the entire rat brain. Given that there are regional differences in insulin uptake and signaling, additional studies are warrented.

Second, the pooled hippocampal microvessels from high-fat diet fed mice also revealed impaired signaling following insulin stimulation (Figure 1). This is interesting, as the inability to stimulate insulin receptors in brain microvascular endothelial cells can decrease blood-brain barrier tight-gap junctions [ $\underline{46}, \underline{47}]$. Moreover, both models of insulin receptor endothelial knock-out [그] and a high-fat diet can decrease insulin signaling in the brain of mice [ㅍ]. In light of our data, this suggests a relationship between barrier insulin receptor dysfunction and underlying brain processes.

Isolated microvessels contain multiple cell types such as endothelial cells, astrocytic end-feet, pericyte or vascular smooth muscle cells [부]. Our goal was to better understand the receptor signaling and expression dynamics specifically in endothelial cells. We reinforce our findings from Figure 1 by moving our studies to in vitro models, demonstrating 
that both primary C57BL/6 mouse brain microvascular endothelial cells (MBECs) and human cerebral microvascular endothelial cells (HBEC-5is) treated with experimental hyperinsulinemia display increases in total insulin receptor expression compared to untreated cells (Figure 2). This hyperinsulinemia-induced change occurred in tandem with increased serine phosphorylation of the insulin receptor substrate (IRS) 1 (Figure 2), a sign of impaired insulin signaling $\underline{29}$. This indicates that hyperinsulinemia is sufficient to impair insulin receptor dysfunction in endothelial cells.

Insulin receptor protein expression and IRS ${ }_{1}$ post-translational modifications are not the only way insulin receptor signaling is modified. There are two receptor variants that differ in the inclusion (variant B, IR-B) and exclusion (variant A, IR-A) of exon 11 in mRNA processing [ [0] $]$. Here, we provide novel evidence of a shift in insulin receptor splice variation in MBECs treated with hyperinsulinemia. Our data reveals a decrease in IR-A levels and IR-A/ IR-B ratios relative to non-hyperinsulinemic controls (Figure 3). Interesting IR$A$ and IR-B differ in their insulin binding kinetics and internalization rates [ $\underline{51}$ ]. Following ligand binding, insulin receptor internalization propagates signal transduction [52]. The IR-A isoform has been previously described as having increased internalization capacity relative to IR-B [ 50], however the splice variant expression response has yet to be determined in the brain microvasculature during hyperinsulinemia. Hence, we sought to assess this further by exploring the impact of hyperinsulinemia on insulin receptor internalization.

Beyond splice variation, the insulin receptor internalization process also relies on key autophosphorylation sites along the $\beta$-subunit, including tyrosines 1150 and 1151 (splice variant "B" numbering) $[\underline{25}, \underline{26}]$, which in turn initiates downstream receptor signaling effectors, leading to phospho-activation of protein kinase B (AKT) [이] . While other tissues such as the liver demonstrate decreased membrane-associated insulin receptor concentrations in models of diabetes [드] , we present a unique finding that our experimentally hyperinsulinemic MBECs demonstrate increased membrane insulin receptor levels (Figure 4). Furthermore, these receptors did not internalize (Supp. Figure 1), nor did they activate downstream signaling cascades following stimulation (Figure 4). All of this coincided with reduced tyrosine autophosphorylation after 15 minutes of stimulation in our model (Figure 4), suggesting that some phosphatase feedback may be enhanced during hyperinsulinemia.

The PTP1B protein is a major regulator of receptor tyrosine activity of the insulin receptor. Recent studies have demonstrated that selective inhibition of PTP1B via Claramine can facilitate tyrosine mediated insulin receptor signaling in vitro [54]. Given our findings of perturbed tyrosine-mediated signaling in hyperinsulinemic cells, we explored whether 30 minutes of Claramine pretreatment prior to stimulation could rescue signaling and internalization. Hyperinsulinemic cells that are treated with Claramine prior to insulin stimulation exhibit restored downstream signaling (Figure 5) and receptor internalization (Figure 5) over baseline. This aligns with previous reports of increased insulin uptake in endothelial cells [이] and improved peripheral insulin sensitivity in vivo [드] following PTP1B inhibition. Further research into the efficacy of Claramine as a brain microvascular endothelial cell insulin receptor sensitizer is warranted, however these results indicate potential for PTP1B inhibition in alleviating insulin receptor dysfunction at this unique microvascular barrier.

In conclusion, we provide data which supports the notion that hyperinsulinemia impacts insulin receptor presentation, internalization, and signaling within brain microvascular endothelial cells in vivo and in vitro. We also offer a potential mechanism to reverse the impact of hyperinsulinemia on insulin receptor dysfunction with Claramine, a potent PTP1B inhibitor. Obesity is a varied disorder with multiple complications including dyslipidemia, atherosclerosis, and systemic inflammation, all of which may confound our findings; thus, we acknowledge the focus on hyperinsulinemia is limited in scope. However, we believe our findings support our claim that hyperinsulinemia plays a significant role in obesity-induced dysfunction. It would be important to follow up these studies in vivo with PTP1B inhibition to assess whether there is functional recovery of the brain 
microvascular endothelial cells in a high-fat diet model. It will also be prudent to understand whether receptor splice variation occurs in the high-fat model. Finally, next steps will be taken to further understand the greater endocytic machinery of the brain endothelial cell, as this has implications beyond insulin receptor functionality which include transcytosis of circulating materials into the brain, as well as functional control over vascular tone and barrier formation.

Supplementary Materials: The following are available online at www.mdpi.com/xxx/s1

Figure S1: Hyperinsulinemic insulin receptors fail to internalize post-stimulation. (A) Representative immunoblots and (B) densitometry analysis of internalized insulin receptors (InsR- $\beta$ ) following $0,5,15$, and 30 minutes of stimulation in mouse brain microvascular endothelial cells that are untreated (Non-HI) or incubated in experimental hyperinsulinemic conditions for 12 hours (HI) Data was analyzed via two-way ANOVA with Tukey's multiple comparisons, $n=6-8$ / group, $N=4$; $* \mathrm{p}<0.05$. All images were normalized to total gel protein concentrations shown as "Stain Free Gel", calculated in Image Lab software per the manufacturer's instructions. Line down center of immunoblot indicates a break in the blot; images are from the same immunoblot for each figure and received identical formatting. Data from 15 minutes post-stimulation is same as Figure 4 non-HI and $\mathrm{HI}$.

Author Contributions: Conceptualization, L.S.W, C.S-R.; Methodology, L.S.W, C.S-R.; Formal Analysis, L.S.W.; Investigation, L.S.W., A.W., G.S., T.L.; Resources, C.S-R.; Data Curation, L.S.W, C.S-R.; Writing - Original Draft Preparation, L.S.W.; Writing - Review \& Editing, B.W.R., A.W., G.S., T.L., C.S-R.; Visualization, C.S-R.; Supervision, C.S-R.; Project Administration, C.S-R.; Funding Acquisition, C.S-R.

Funding: This research was funded by the National Institutes of Health (NHLBI 4T32HL007260-40 to L.S.W. and BWR; NINDS 5K01NS079461, NINDS 1R01NS099595, and NIGMS P20GM109040 to C. S-R.) and the Alzheimer's Association (AARGD-16-440893 to C.S-R.). The funding bodies played no role in the design of the study, nor the collection, analysis, and interpretation of data, nor in writing the manuscript.

Institutional Review Board Statement: The study was conducted in accordance with the university guidelines, state and federal regulations, and the standards of the "Guide for the Care and Use of Laboratory Animals." All protocols/ procedures were approved by the Medical University of South Carolina Institutional Animal Care and Use Committee.

Informed Consent Statement: Not applicable.

Data Availability Statement: The data that support the findings of this study are available from the corresponding author upon reasonable request.

Acknowledgments: The authors wish to acknowledge Janet Boggs and Stephanie DiLucia for technical assistance and support.

Conflicts of Interest: L.S.W. is currently employee of Intra-Cellular Therapies, Inc., however his employment played no role in the presentation of these findings. All other authors declare that they have no conflicting interests. The funders had no role in the design of the study; in the collection, analyses, or interpretation of data; in the writing of the manuscript, or in the decision to publish the results".

\section{References}

1. Toth, P.; Tarantini, S.; Csiszar, A.; Ungvari, Z. Functional vascular contributions to cognitive impairment and dementia: mechanisms and consequences of cerebral autoregulatory dysfunction, endothelial impairment, and neurovascular uncoupling in aging. American journal of physiology. Heart and circulatory physiology 2017, 312, H1-H20, doi:10.1152/ajpheart.00581.2016.

2. Jiang, S.Z.; Lu, W.; Zong, X.F.; Ruan, H.Y.; Liu, Y. Obesity and hypertension. Exp Ther Med 2016, 12, 2395-2399, doi:10.3892/etm.2016.3667.

3. Wahl, D.; Solon-Biet, S.M.; Cogger, V.C.; Fontana, L.; Simpson, S.J.; Le Couteur, D.G.; Ribeiro, R.V. Aging, lifestyle and dementia. Neurobiol Dis 2019, 130, 104481, doi:10.1016/j.nbd.2019.104481. 
4. Raji, C.A.; Ho, A.J.; Parikshak, N.N.; Becker, J.T.; Lopez, O.L.; Kuller, L.H.; Hua, X.; Leow, A.D.; Toga, A.W.; Thompson, P.M. Brain structure and obesity. Hum Brain Mapp 2010, 31, 353-364, doi:10.1002/hbm.20870.

5. Buie, J.J.; Watson, L.S.; Smith, C.J.; Sims-Robinson, C. Obesity-related cognitive impairment: The role of endothelial dysfunction. Neurobiol Dis 2019, 132, 104580, doi:10.1016/j.nbd.2019.104580.

6. Hales, C.M.; Carroll, M.D.; Fryar, C.D.; Ogden, C.L. Prevalence of Obesity and Severe Obesity Among Adults: United States, 2017-2018. NCHS Data Brief 2020, 1-8.

7. Kopp, W. How Western Diet And Lifestyle Drive The Pandemic Of Obesity And Civilization Diseases. Diabetes Metab Syndr Obes 2019, 12, 2221-2236, doi:10.2147/DMSO.S216791.

8. Watson, L.S.; Stone, T.D.; Williams, D.; Williams, A.S.; Sims-Robinson, C. High-Fat diet impairs tactile discrimination memory in the mouse. Behav Brain Res 2020, 10.1016/j.bbr.2019.112454, 112454, doi:10.1016/j.bbr.2019.112454.

9. Sims-Robinson, C.; Bakeman, A.; Bruno, E.; Jackson, S.; Glasser, R.; Murphy, G.G.; Feldman, E.L. Dietary Reversal Ameliorates Short- and Long-Term Memory Deficits Induced by High-fat Diet Early in Life. PLoS One 2016, 11, e0163883, doi:10.1371/journal.pone.0163883.

10. Williams, A.; Lowry, T.; Sims-Robinson, C. The development of a cognitive rehabilitation task for mice. Neurobiol Learn Mem 2020, 175, 107296, doi:10.1016/j.nlm.2020.107296.

11. Kasper, J.M.; Milton, A.J.; Smith, A.E.; Laezza, F.; Taglialatela, G.; Hommel, J.D.; Abate, N. Cognitive deficits associated with a high-fat diet and insulin resistance are potentiated by overexpression of ecto-nucleotide pyrophosphatase phosphodiesterase-1. Int J Dev Neurosci 2018, 64, 48-53, doi:10.1016/j.ijdevneu.2017.03.011.

12. Shanik, M.H.; Xu, Y.; Skrha, J.; Dankner, R.; Zick, Y.; Roth, J. Insulin resistance and hyperinsulinemia: is hyperinsulinemia the cart or the horse? Diabetes Care 2008, 31 Suppl 2, S262-268, doi:10.2337/dc08-s264.

13. Sims-Robinson, C.; Kim, B.; Rosko, A.; Feldman, E.L. How does diabetes accelerate Alzheimer disease pathology? Nat Rev Neurol 2010, 6, 551-559, doi:10.1038/nrneurol.2010.130.

14. Yang, A.C.; Stevens, M.Y.; Chen, M.B.; Lee, D.P.; Stahli, D.; Gate, D.; Contrepois, K.; Chen, W.; Iram, T.; Zhang, L., et al. Physiological blood-brain transport is impaired with age by a shift in transcytosis. Nature 2020, 583, 425-430, doi:10.1038/s41586020-2453-z.

15. Daneman, R.; Prat, A. The blood-brain barrier. Cold Spring Harb Perspect Biol 2015, 7, a020412, doi:10.1101/cshperspect.a020412.

16. Redzic, Z. Molecular biology of the blood-brain and the blood-cerebrospinal fluid barriers: similarities and differences. Fluids Barriers CNS 2011, 8, 3, doi:10.1186/2045-8118-8-3.

17. Blumling Iii, J.P.; Silva, G.A. Targeting the brain: advances in drug delivery. Current pharmaceutical biotechnology 2012, 13, 2417-2426.

18. Pardridge, W.M. Re-engineering biopharmaceuticals for delivery to brain with molecular Trojan horses. Bioconjugate chemistry 2008, 19, 1327-1338, doi:10.1021/bc800148t.

19. Pardridge, W.M.; Boado, R.J. Reengineering biopharmaceuticals for targeted delivery across the blood-brain barrier. Methods in enzymology 2012, 503, 269-292, doi:10.1016/B978-0-12-396962-0.00011-2.

20. Gray, S.M.; Aylor, K.W.; Barrett, E.J. Unravelling the regulation of insulin transport across the brain endothelial cell. Diabetologia 2017, 60, 1512-1521, doi:10.1007/s00125-017-4285-4.

21. Meijer, R.I.; Gray, S.M.; Aylor, K.W.; Barrett, E.J. Pathways for insulin access to the brain: the role of the microvascular endothelial cell. Am J Physiol Heart Circ Physiol 2016, 311, H1132-H1138, doi:10.1152/ajpheart.00081.2016.

22. White, M.F. The insulin signalling system and the IRS proteins. Diabetologia 1997, 40 Suppl 2, S2-17, doi:10.1007/s001250051387.

23. Cheatham, B.; Kahn, C.R. Insulin action and the insulin signaling network. Endocrine reviews 1995, 16, 117-142, doi:10.1210/edrv-16-2-117.

24. Draznin, B. Molecular mechanisms of insulin resistance: serine phosphorylation of insulin receptor substrate-1 and increased expression of p85alpha: the two sides of a coin. Diabetes 2006, 55, 2392-2397, doi:10.2337/db06-0391.

25. Backer, J.M.; Shoelson, S.E.; Haring, E.; White, M.F. Insulin receptors internalize by a rapid, saturable pathway requiring receptor autophosphorylation and an intact juxtamembrane region. J Cell Biol 1991, 115, 1535-1545, doi:10.1083/jcb.115.6.1535.

26. Carpentier, J.L. Insulin receptor internalization: molecular mechanisms and physiopathological implications. Diabetologia 1994, 37 Suppl 2, S117-124, doi:10.1007/BF00400835.

27. Fagerholm, S.; Ortegren, U.; Karlsson, M.; Ruishalme, I.; Stralfors, P. Rapid insulin-dependent endocytosis of the insulin receptor by caveolae in primary adipocytes. PLoS One 2009, 4, e5985, doi:10.1371/journal.pone.0005985.

28. Murphy, J.E.; Padilla, B.E.; Hasdemir, B.; Cottrell, G.S.; Bunnett, N.W. Endosomes: a legitimate platform for the signaling train. Proc Natl Acad Sci U S A 2009, 106, 17615-17622, doi:10.1073/pnas.0906541106.

29. Kublaoui, B.; Lee, J.; Pilch, P.F. Dynamics of signaling during insulin-stimulated endocytosis of its receptor in adipocytes. J Biol Chem 1995, 270, 59-65, doi:10.1074/jbc.270.1.59.

30. Wang, H.; Wang, A.X.; Liu, Z.; Barrett, E.J. Insulin signaling stimulates insulin transport by bovine aortic endothelial cells. Diabetes 2008, 57, 540-547, doi:10.2337/db07-0967.

31. Tanti, J.F.; Jager, J. Cellular mechanisms of insulin resistance: role of stress-regulated serine kinases and insulin receptor substrates (IRS) serine phosphorylation. Curr Opin Pharmacol 2009, 9, 753-762, doi:10.1016/j.coph.2009.07.004. 
32. Goldstein, B.J. Protein-tyrosine phosphatase 1B (PTP1B): a novel therapeutic target for type 2 diabetes mellitus, obesity and related states of insulin resistance. Curr Drug Targets Immune Endocr Metabol Disord 2001, 1, 265-275, doi:10.2174/1568008013341163.

33. Howland, S.W.; Gun, S.Y.; Claser, C.; Poh, C.M.; Renia, L. Measuring antigen presentation in mouse brain endothelial cells ex vivo and in vitro. Nat Protoc 2015, 10, 2016-2026, doi:10.1038/nprot.2015.129.

34. Ruck, T.; Bittner, S.; Epping, L.; Herrmann, A.M.; Meuth, S.G. Isolation of primary murine brain microvascular endothelial cells. Journal of visualized experiments : JoVE 2014, 10.3791/52204, e52204, doi:10.3791/52204.

35. Zhao, N.; Liu, C.C.; Van Ingelgom, A.J.; Martens, Y.A.; Linares, C.; Knight, J.A.; Painter, M.M.; Sullivan, P.M.; Bu, G. Apolipoprotein E4 Impairs Neuronal Insulin Signaling by Trapping Insulin Receptor in the Endosomes. Neuron 2017, 96, 115129 e115, doi:10.1016/j.neuron.2017.09.003.

36. Pischedda, F.; Szczurkowska, J.; Cirnaru, M.D.; Giesert, F.; Vezzoli, E.; Ueffing, M.; Sala, C.; Francolini, M.; Hauck, S.M.; Cancedda, L., et al. A cell surface biotinylation assay to reveal membrane-associated neuronal cues: Negr1 regulates dendritic arborization. Mol Cell Proteomics 2014, 13, 733-748, doi:10.1074/mcp.M113.031716.

37. Kim, B.; Sullivan, K.A.; Backus, C.; Feldman, E.L. Cortical neurons develop insulin resistance and blunted Akt signaling: a potential mechanism contributing to enhanced ischemic injury in diabetes. Antioxid Redox Signal 2011, 14, 1829-1839, doi:10.1089/ars.2010.3816.

38. Rowzee, A.M.; Ludwig, D.L.; Wood, T.L. Insulin-like growth factor type 1 receptor and insulin receptor isoform expression and signaling in mammary epithelial cells. Endocrinology 2009, 150, 3611-3619, doi:10.1210/en.2008-1473.

39. Israel, P.A.; Park, C.R.; Schwartz, M.W.; Green, P.K.; Sipols, A.J.; Woods, S.C.; Porte, D., Jr.; Figlewicz, D.P. Effect of dietinduced obesity and experimental hyperinsulinemia on insulin uptake into CSF of the rat. Brain Res Bull 1993, 30, 571-575, doi:10.1016/0361-9230(93)90084-o.

40. Kaiyala, K.J.; Prigeon, R.L.; Kahn, S.E.; Woods, S.C.; Schwartz, M.W. Obesity induced by a high-fat diet is associated with reduced brain insulin transport in dogs. Diabetes 2000, 49, 1525-1533, doi:10.2337/diabetes.49.9.1525.

41. Rhea, E.M.; Rask-Madsen, C.; Banks, W.A. Insulin transport across the blood-brain barrier can occur independently of the insulin receptor. The Journal of physiology 2018, 596, 4753-4765, doi:10.1113/JP276149.

42. De Meyts, P. The Insulin Receptor and Its Signal Transduction Network. In Endotext, Feingold, K.R., Anawalt, B., Boyce, A., Chrousos, G., de Herder, W.W., Dungan, K., Grossman, A., Hershman, J.M., Hofland, J., Kaltsas, G., et al., Eds. South Dartmouth (MA), 2000.

43. Katakam, P.V.; Domoki, F.; Lenti, L.; Gaspar, T.; Institoris, A.; Snipes, J.A.; Busija, D.W. Cerebrovascular responses to insulin in rats. J Cereb Blood Flow Metab 2009, 29, 1955-1967, doi:10.1038/jcbfm.2009.177.

44. Sims-Robinson, C.; Bakeman, A.; Glasser, R.; Boggs, J.; Pacut, C.; Feldman, E.L. The role of endoplasmic reticulum stress in hippocampal insulin resistance. Exp Neurol 2016, 277, 261-267, doi:10.1016/j.expneurol.2016.01.007.

45. Janson, J.; Laedtke, T.; Parisi, J.E.; O'Brien, P.; Petersen, R.C.; Butler, P.C. Increased risk of type 2 diabetes in Alzheimer disease. Diabetes 2004, 53, 474-481, doi:10.2337/diabetes.53.2.474.

46. Beard, R.S., Jr.; Hoettels, B.A.; Meegan, J.E.; Wertz, T.S.; Cha, B.J.; Yang, X.; Oxford, J.T.; Wu, M.H.; Yuan, S.Y. AKT2 maintains brain endothelial claudin-5 expression and selective activation of IR/AKT2/FOXO1-signaling reverses barrier dysfunction. J Cereb Blood Flow Metab 2020, 40, 374-391, doi:10.1177/0271678X18817512.

47. Konishi, M.; Sakaguchi, M.; Lockhart, S.M.; Cai, W.; Li, M.E.; Homan, E.P.; Rask-Madsen, C.; Kahn, C.R. Endothelial insulin receptors differentially control insulin signaling kinetics in peripheral tissues and brain of mice. Proceedings of the National Academy of Sciences of the United States of America 2017, 114, E8478-E8487, doi:10.1073/pnas.1710625114.

48. Ruegsegger, G.N.; Vanderboom, P.M.; Dasari, S.; Klaus, K.A.; Kabiraj, P.; McCarthy, C.B.; Lucchinetti, C.F.; Nair, K.S. Exercise and metformin counteract altered mitochondrial function in the insulin-resistant brain. JCI Insight 2019, 4, doi:10.1172/jci.insight.130681.

49. Paraiso, H.C.; Wang, X.; Kuo, P.C.; Furnas, D.; Scofield, B.A.; Chang, F.L.; Yen, J.H.; Yu, I.C. Isolation of Mouse Cerebral Microvasculature for Molecular and Single-Cell Analysis. Front Cell Neurosci 2020, 14, 84, doi:10.3389/fncel.2020.00084.

50. Malakar, P.; Chartarifsky, L.; Hija, A.; Leibowitz, G.; Glaser, B.; Dor, Y.; Karni, R. Insulin receptor alternative splicing is regulated by insulin signaling and modulates beta cell survival. Sci Rep 2016, 6, 31222, doi:10.1038/srep31222.

51. Kellerer, M.; Lammers, R.; Ermel, B.; Tippmer, S.; Vogt, B.; Obermaier-Kusser, B.; Ullrich, A.; Haring, H.U. Distinct alpha-subunit structures of human insulin receptor $A$ and $B$ variants determine differences in tyrosine kinase activities. Biochemistry 1992, 31, 4588-4596, doi:10.1021/bi00134a008.

52. Di Guglielmo, G.M.; Drake, P.G.; Baass, P.C.; Authier, F.; Posner, B.I.; Bergeron, J.J. Insulin receptor internalization and signalling. Mol Cell Biochem 1998, 182, 59-63.

53. Soll, A.H.; Kahn, C.R.; Neville, D.M., Jr. Insulin binding to liver plasm membranes in the obese hyperglycemic (ob/ob) mouse. Demonstration of a decreased number of functionally normal receptors. J Biol Chem 1975, 250, 4702-4707.

54. Qin, Z.; Pandey, N.R.; Zhou, X.; Stewart, C.A.; Hari, A.; Huang, H.; Stewart, A.F.; Brunel, J.M.; Chen, H.H. Functional properties of Claramine: a novel PTP1B inhibitor and insulin-mimetic compound. Biochem Biophys Res Commun 2015, 458, 2127, doi:10.1016/j.bbrc.2015.01.040.

55. Dodd, G.T.; Xirouchaki, C.E.; Eramo, M.; Mitchell, C.A.; Andrews, Z.B.; Henry, B.A.; Cowley, M.A.; Tiganis, T. Intranasal Targeting of Hypothalamic PTP1B and TCPTP Reinstates Leptin and Insulin Sensitivity and Promotes Weight Loss in Obesity. Cell Rep 2019, 28, 2905-2922 e2905, doi:10.1016/j.celrep.2019.08.019. 